Louisiana State University

LSU Digital Commons

2-10-2014

\title{
Dust production and particle acceleration in supernova 1987a revealed with Alma
}

\author{
R. Indebetouw \\ University of Virginia \\ M. Matsuura \\ University College London \\ E. Dwek \\ NASA Goddard Space Flight Center \\ G. Zanardo \\ The University of Western Australia \\ M. J. Barlow \\ University College London
}

See next page for additional authors

Follow this and additional works at: https://digitalcommons.Isu.edu/physics_astronomy_pubs

\section{Recommended Citation}

Indebetouw, R., Matsuura, M., Dwek, E., Zanardo, G., Barlow, M., Baes, M., Bouchet, P., Burrows, D., Chevalier, R., Clayton, G., Fransson, C., Gaensler, B., Kirshner, R., Lakićević, M., Long, K., Lundqvist, P., MartíVidal, I., Marcaide, J., McCray, R., Meixner, M., Ng, C., Park, S., Sonneborn, G., Staveley-Smith, L., Vlahakis, C., \& Van Loon, J. (2014). Dust production and particle acceleration in supernova 1987a revealed with Alma. Astrophysical Journal Letters, 782 (1) https://doi.org/10.1088/2041-8205/782/1/L2

This Article is brought to you for free and open access by the Department of Physics \& Astronomy at LSU Digital Commons. It has been accepted for inclusion in Faculty Publications by an authorized administrator of LSU Digital Commons. For more information, please contact ir@lsu.edu. 


\section{Authors}

R. Indebetouw, M. Matsuura, E. Dwek, G. Zanardo, M. J. Barlow, M. Baes, P. Bouchet, D. N. Burrows, R. Chevalier, G. C. Clayton, C. Fransson, B. Gaensler, R. Kirshner, M. Lakićević, K. S. Long, P. Lundqvist, I. Martí-Vidal, J. Marcaide, R. McCray, M. Meixner, C. Y. Ng, S. Park, G. Sonneborn, L. Staveley-Smith, C. Vlahakis, and J. Van Loon 


\title{
DUST PRODUCTION AND PARTICLE ACCELERATION IN SUPERNOVA 1987A REVEALED WITH ALMA
}

\author{
R. Indebetouw ${ }^{1,2}$, M. Matsuura ${ }^{3}$, E. DweK ${ }^{4}$, G. Zanardo ${ }^{5}$, M. J. Barlow ${ }^{3}$, M. Baes ${ }^{6}$, P. Bouchet ${ }^{7}$, D. N. Burrows $^{8}$, \\ R. Chevalier ${ }^{1}$, G. C. Clayton ${ }^{9}$, C. Fransson ${ }^{10}$, B. Gaensler ${ }^{11,12}$, R. Kirshner ${ }^{13}$, M. Lakićević ${ }^{14}$, K. S. Long ${ }^{15}$, \\ P. Lundqvist ${ }^{10}$, I. Martí-Vidal ${ }^{16}$, J. Marcaide $^{17}$, R. McCray ${ }^{18}$, M. MeiXner ${ }^{15}, 19$, C.-Y. NG ${ }^{20}$, S. ParK $^{21}$, G. Sonneborn $^{15}$, \\ L. Staveley-Smith ${ }^{5,11}$, C. Vlahakis ${ }^{22}$, AND J. VAN LOON ${ }^{14}$ \\ ${ }^{1}$ Department of Astronomy, University of Virginia, PO Box 400325, Charlottesville, VA 22904, USA; remy@ virginia.edu \\ ${ }^{2}$ National Radio Astronomy Observatory, 520 Edgemont Rd, Charlottesville, VA 22903, USA \\ ${ }^{3}$ Department of Physics and Astronomy, University College London, Gower Street, London WC1E 6BT, UK \\ ${ }^{4}$ NASA Goddard Space Flight Center, 8800 Greenbelt Road, Greenbelt, MD 20771, USA \\ ${ }^{5}$ International Centre for Radio Astronomy Research (ICRAR), University of Western Australia, Crawley, WA 6009, Australia \\ ${ }^{6}$ Sterrenkundig Observatorium, Universiteit Gent, Krijgslaan 281 S9, B-9000 Gent, Belgium \\ ${ }^{7}$ CEA-Saclay, F-91191 Gif-sur-Yvette, France \\ ${ }^{8}$ Department of Astronomy \& Astrophysics, The Pennsylvania State University, University Park, PA 16802, USA \\ ${ }^{9}$ Department of Physics and Astronomy, Louisiana State University, Baton Rouge, LA 70803, USA \\ ${ }^{10}$ Department of Astronomy and the Oskar Klein Centre, Stockholm University, AlbaNova, SE-106 91 Stockholm, Sweden \\ ${ }^{11}$ Australian Research Council Centre of Excellence for All-sky Astrophysics (CAASTRO) \\ 12 Sydney Institute for Astronomy, School of Physics, The University of Sydney, NSW 2006, Australia \\ ${ }^{13}$ Harvard-Smithsonian Center for Astrophysics, 60 Garden St., Cambridge, MA 02138, USA \\ ${ }^{14}$ Lennard-Jones Laboratories, Keele University, ST5 5BG, UK \\ ${ }^{15}$ Space Telescope Science Institute, 3700 San Martin Drive, Baltimore, MD 21218, USA \\ ${ }^{16}$ Department of Earth and Space Sciences, Chalmers University of Technology, Onsala Space Observatory, SE-43992 Onsala, Sweden \\ ${ }^{17}$ Universidad de Valencia, C/Dr. Moliner 50, E-46100 Burjassot, Spain \\ ${ }^{18}$ Department of Astrophysical and Planetary Sciences, University of Colorado at Boulder, UCB 391, Boulder, CO 80309, USA \\ ${ }^{19}$ The Johns Hopkins University, Department of Physics and Astronomy, 366 Bloomberg Center, 3400 N. Charles Street, Baltimore, MD 21218, USA \\ ${ }^{20}$ Department of Physics, The University of Hong Kong, Pokfulam Road, Hong Kong \\ ${ }^{21}$ Department of Physics, University of Texas at Arlington, 108 Science Hall, Box 19059, Arlington, TX 76019, USA \\ 22 Joint ALMA Observatory/European Southern Observatory, Alonso de Cordova 3107, Vitacura, Santiago, Chile \\ Received 2013 November 12; accepted 2013 December 13; published 2014 January 17
}

\begin{abstract}
Supernova (SN) explosions are crucial engines driving the evolution of galaxies by shock heating gas, increasing the metallicity, creating dust, and accelerating energetic particles. In 2012 we used the Atacama Large Millimeter/ Submillimeter Array to observe SN 1987A, one of the best-observed supernovae since the invention of the telescope. We present spatially resolved images at $450 \mu \mathrm{m}, 870 \mu \mathrm{m}, 1.4 \mathrm{~mm}$, and $2.8 \mathrm{~mm}$, an important transition wavelength range. Longer wavelength emission is dominated by synchrotron radiation from shock-accelerated particles, shorter wavelengths by emission from the largest mass of dust measured in a supernova remnant $\left(>0.2 M_{\odot}\right)$. For the first time we show unambiguously that this dust has formed in the inner ejecta (the cold remnants of the exploded star's core). The dust emission is concentrated at the center of the remnant, so the dust has not yet been affected by the shocks. If a significant fraction survives, and if SN 1987A is typical, supernovae are important cosmological dust producers.
\end{abstract}

Key words: galaxies: ISM - ISM: supernova remnants - Magellanic Clouds - supernovae: individual (1987A)

\section{INTRODUCTION}

Supernova (SN) 1987A in the Large Magellanic Cloud was the closest supernova explosion to Earth $(50 \mathrm{kpc})$ observed since Kepler's SN1604AD, making it a unique target to study supernova and supernova remnant physics. Supernovae are thought to be one of the most important sources of dust in the universe. Stars synthesize heavy elements, and after they explode, rapid expansion and radiation cause the ejected gas to cool rapidly, allowing refractory elements to condense into dust grains. Dust masses have been measured in over 20 supernovae and supernova remnants (Gall et al. 2011); most contain $10^{-6}-10^{-3} M_{\odot}$ of warm dust, far below predictions of 0.1-1.0 $M_{\odot}$ (Todini \& Ferrara 2001; Nozawa et al. 2003; Cherchneff \& Dwek 2010). One of the few exceptions is SN 1987A-far infrared photometry $(100-350 \mu \mathrm{m})$ with the Herschel Space Observatory in 2011 implied 0.4-0.7 $M_{\odot}$ of dust at 20-26 K (Matsuura et al. 2011). Measured 24 years after the explosion, that dust mass is significantly higher than the $10^{-4} M_{\odot}$ reported from infrared measurements one to two years after the explosion (Wooden et al. 1993; Bouchet et al.
2004). While the Herschel result supports theoretical models of significant dust production, the discrepancy with prior results caused many to question whether all of the dust detected in 2011 was in fact produced in the supernova. SN 1987A's progenitor star could have created a massive dust shell during its red supergiant phase. Mid-infrared (MIR) photometry of SN 1987A in 2003 (Bouchet et al. 2004) and spectroscopy in 2005 (Dwek et al. 2010) only found $10^{-5} M_{\odot}$ of warm progenitor dust. However, observations of Galactic evolved stars with masses bracketing that of SN 1987A's progenitor reveal between 0.01 and $0.4 M_{\odot}$ of dust (the Egg Nebula, Jura et al. 2000 and Eta Carina, Gomez et al. 2010), so if only a minor warm component of the progenitor dust was detected in the MIR, a large cold progenitor dust mass detected for the first time by Herschel is plausible. Herschel had insufficient spatial resolution to distinguish between the ejecta, shocked progenitor wind, and nearby interstellar material.

Resolved observations of SN 1987A in the submillimeter regime are required to establish the location of the emission measured by Herschel. Multi-wavelength resolved images are required to determine its physical origin, and such images can 
be used to simultaneously study shock particle acceleration. Twenty-five years after the explosion, SN 1987A's blast wave had propagated to a radius of $7 \times 10^{17} \mathrm{~cm}\left(0^{\prime} \cdot 9\right.$ on the sky), shocking material lost from the progenitor star (Crotts \& Heathcote 2000). Electrons accelerated by the shocks produce a bright shell or torus of synchrotron radiation at centimeter wavelengths (Potter et al. 2009; Ng et al. 2013). The shocks are interacting with a dense equatorial ring tilted 43 degrees from the line-of-sight (Tziamtzis et al. 2011), dominating the emission at X-ray, optical to thermal infrared $(<30 \mu \mathrm{m})$ wavelengths (Bouchet et al. 2004; Dwek et al. 2010). Interior to the shock and ring are the remnants of the star's metalrich core, referred to hereafter as the inner ejecta. A supernova explosion can also leave behind a black hole or a neutron star. The observed neutrino burst implies that a neutron star must have at least temporarily formed in SN 1987A (McCray 1993), but it has yet to be detected. If the neutron star energizes a pulsar wind nebula (PWN), its emission would most likely have a spectrum with power-law spectral index of -0.3 to 0 (Gaensler \& Slane 2006), flatter than synchrotron emission from the shock, perhaps detectable at millimeter wavelengths or even contributing to the Herschel far-infrared emission. Previous observations at $10 \mathrm{~mm}$ (Potter et al. 2009), $6.8 \mathrm{~mm}$ (Zanardo et al. 2013), and $3.2 \mathrm{~mm}$ (Lakićević et al. 2012b) tentatively detected such excess emission at the remnant's center, with large uncertainties from image deconvolution; high spatial resolution images from millimeter to submillimeter wavelengths are essential to disentangle the emission mechanisms.

\section{OBSERVATIONS}

The Atacama Large Millimeter/Submillimeter Array (ALMA) observations are executed according to the quality of the weather, and in general the observing requirements are more stringent the higher the frequency of the observation. SN 1987A was observed during 2012 multiple times with $\sim 20$ antennas in configurations containing baselines between $\sim 20 \mathrm{~m}$ and $\sim 400 \mathrm{~m}$. Bands 3,6,7, and $9(2.8 \mathrm{~mm}, 1.4 \mathrm{~mm}, 870 \mu \mathrm{m}$, and 450 $\mu \mathrm{m})$ were observed in approximately chronological order. Band 3: A002/X3c5ee0/X24b (April 5), A002/X3c7a84/X1c (April 6); Band 6: A002/X3c8f66/X352 (April 7), A002/X45f1dd/ Xd13 (July 15), A002/X494155/X8be (August 10); Band 7: A002/X45e2af/X458 (July 14), A002/X4ae797/X776 (August 24); Band 9: A002/X4afc39/X8ce (August 25), A002/ X4b29af/Xd21 (August 27), A002/X535168/X796 (November 5). Each data set was calibrated individually with Common Astronomy Software Applications (casa.nrao.edu). All data in a given wavelength band were then combined for imaging and deconvolution with the "clean" algorithm. Only the channels free of bright $\mathrm{CO}$ and $\mathrm{SiO}$ emission were used $(212.56-213.59 \mathrm{GHz}$ and 100.07-103.91 GHz in Bands 6 and 3). In synthesis imaging the weighting as a function of baseline length can be adjusted to achieve finer spatial resolution at the expense of somewhat higher noise per beam. Briggs weighting was used with "robust" parameters and resulting Gaussian restoring beams as noted in the figures. The Australia Telescope Compact Array (ATCA) images were deconvolved using the maximum entropy algorithm (Zanardo et al. 2013; Lakićević et al. 2012b).

\section{RESULTS}

In Figure 1, we present the first spatially resolved submillimeter continuum observations of SN 1987A, obtained with the ALMA.
We can now separate emission from the torus from that of the inner ejecta at $450 \mu \mathrm{m}, 870 \mu \mathrm{m}$, and $1.4 \mathrm{~mm}$. Decomposition of the torus and inner ejecta was performed in the image plane. The torus was represented with the clean model (collection of point sources or clean components) from deconvolving the ATCA $6.8 \mathrm{~mm}$ image. The inner ejecta were represented by the ALMA Band $9(450 \mu \mathrm{m})$ model. For each band, the image was decomposed into a clean model and residual. The torus or ejecta model, scaled in amplitude, was subtracted from the model, which was then restored with the appropriate Gaussian restoring beam, and the residual image added back in. This method results in minimal noise from the torus or ejecta observation being introduced, merely preserving the noise already present in each original image. The amplitude scaling is varied to minimize the residuals in the subtracted image. The torus and ejecta were also subtracted from each other in the Fourier plane, with consistent results. The torus and ejecta flux densities measured using these independent decompositions were combined to determine the flux densities and uncertainties listed in Table 1.

\section{DISCUSSION}

We discuss the torus first since the physical emission mechanism, synchrotron emission from shock-accelerated particles, is less controversial than the inner ejecta emission mechanism. Images of the torus alone (central ejecta removed) are shown in Figure 2. The eastern side is brighter at all (sub)millimeter wavelengths, and the asymmetry decreases at shorter wavelengths. In contrast, $\mathrm{H} \alpha$ emission (Larsson et al. 2013) from shocks being driven into the equatorial ring, and soft X-ray emission (Helder et al. 2013) from hot plasma behind the shocks is now brighter in the west (Figure 1). All wavelengths shown have brightened with time, and the emission distribution has become geometrically flatter in the equatorial plane, as the shocks interact with the dense ring ( $\mathrm{Ng}$ et al. 2013; Racusin et al. 2009). Asymmetry results from differences in how far that interaction has progressed (e.g., the X-ray asymmetry can be explained by faster shocks in the east; Zhekov et al. 2009).

The integrated spectrum of the torus (Figure 3 ) is fit from $17 \mathrm{~mm}$ to $870 \mu \mathrm{m}$ by a power law with a spectral index $\alpha=-0.8 \pm 0.1\left(F_{v} \propto v^{\alpha}\right)$ and no evident spectral break. This rules out significant synchrotron losses that would steepen the spectrum or contamination from free-free emission that would flatten it. The spectrum is quite steep (few high energy particles), compared to what would be produced by diffusive shock acceleration in the test particle limit, in a single shock at the observed $\sim 2000 \mathrm{~km} \mathrm{~s}^{-1}$ expansion velocity (Helder et al. 2013; $\mathrm{Ng}$ et al. 2013). The shock structure is therefore likely being modified by pressure from the accelerated particles (Ellison et al. 2000) or by an amplified tangled magnetic field (Kirk et al. 1996).

The torus with its simple spectrum can now be removed from the images to isolate the inner ejecta (Figure 4). Inner ejecta emission is well resolved at $450 \mu \mathrm{m}$, with a beam-deconvolved FWHM of $0.3 \pm 0.03$ by $0.16 \pm 0$ '. 05 , or $2.2 \pm 0.2 \times 10^{17} \mathrm{~cm}$ by $1.2 \pm 0.4 \times 10^{17} \mathrm{~cm}$, corresponding to constant expansion velocities of $1350 \pm 150 \mathrm{~km} \mathrm{~s}^{-1}$ by $750 \pm 250 \mathrm{~km} \mathrm{~s}^{-1}$. It is marginally resolved at $870 \mu \mathrm{m}$, constrained by the observations to have $\mathrm{FWHM}<0$ !' 4 , consistent with the $450 \mu \mathrm{m}$ size.

The spectrum of the inner ejecta rises steeply with frequency, inconsistent with PWN emission, but very well modeled by dust. The ALMA and Herschel photometry can be fit with $0.23 \pm 0.05 M_{\odot}$ of amorphous carbon dust at $26 \pm 3 \mathrm{~K}$ 

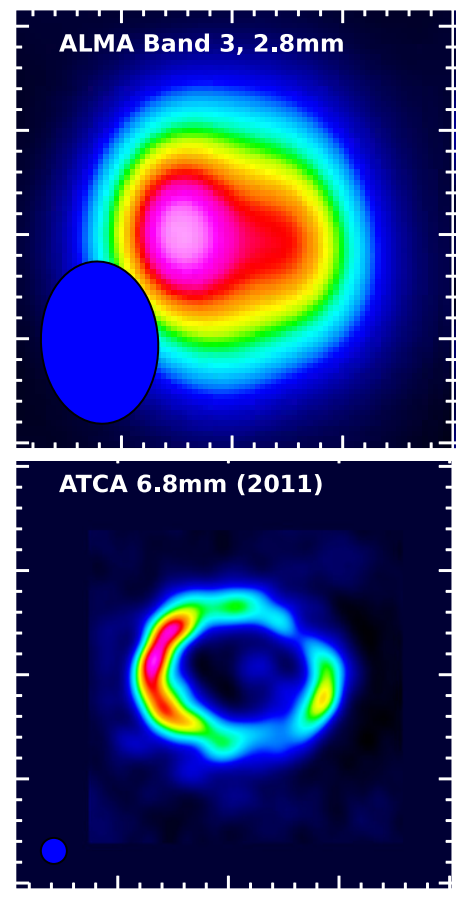
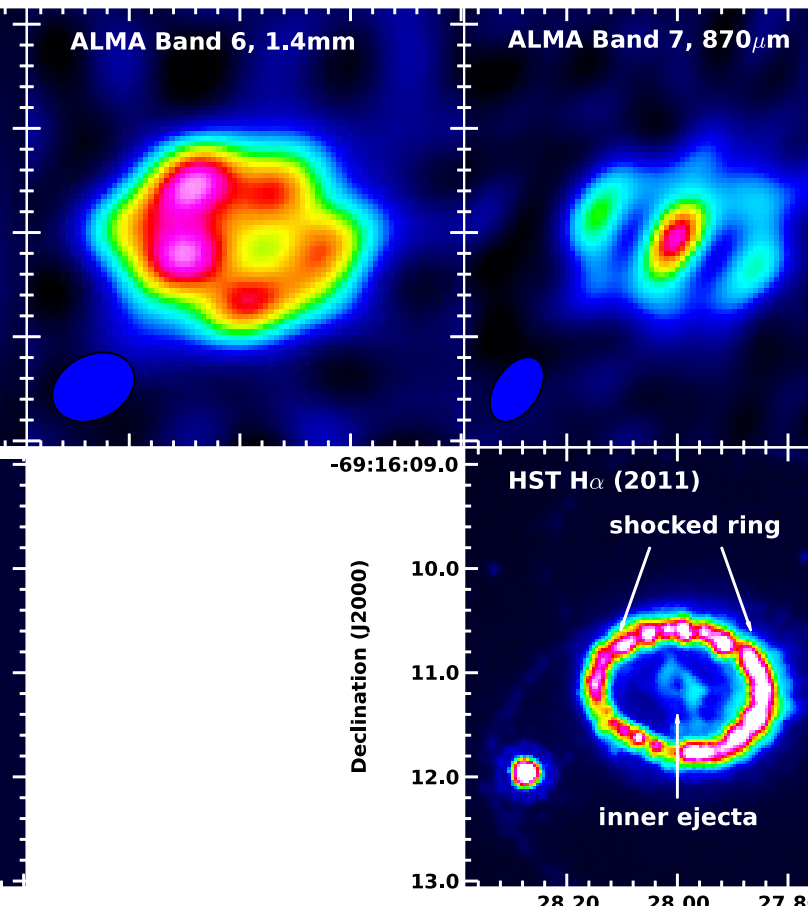

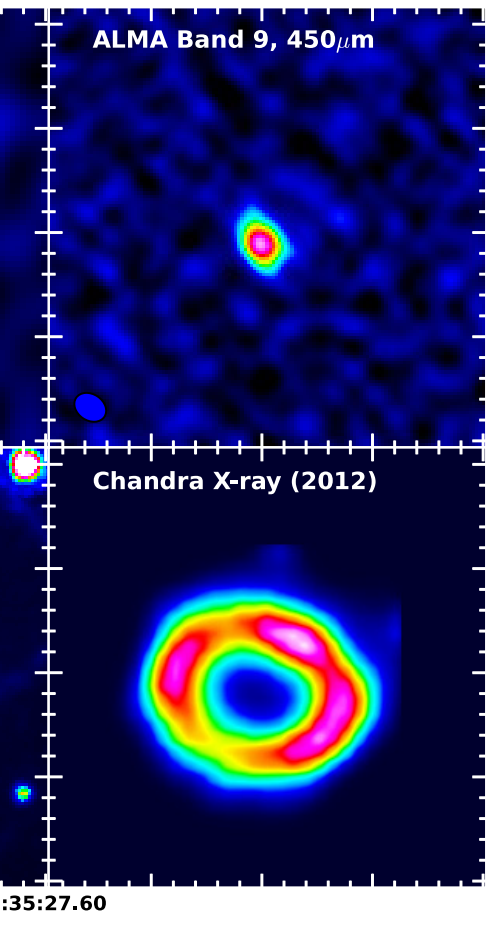

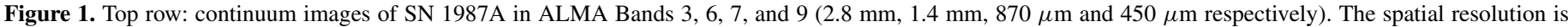

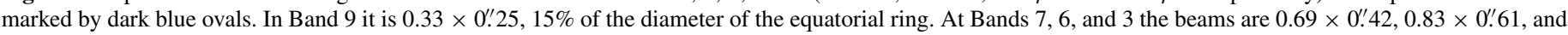

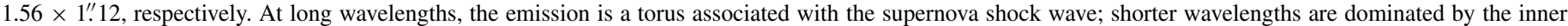

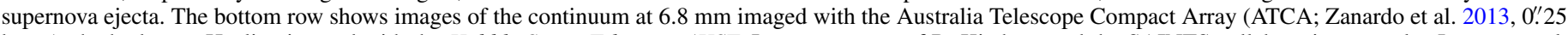

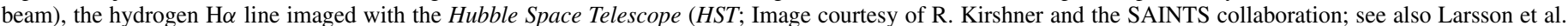
2013), and the soft X-ray emission imaged with the Chandra X-Ray Observatory (Helder et al. 2013).

Table 1

Flux Densities

\begin{tabular}{|c|c|c|c|c|c|c|c|}
\hline Component & $\begin{array}{c}v \\
(\mathrm{GHz})\end{array}$ & $\lambda$ & $\begin{array}{c}F_{v} \\
(\mathrm{mJy})\end{array}$ & Epoch & Telescope & $\begin{array}{c}\text { Angular } \\
\text { Res. }\end{array}$ & Ref. \\
\hline Torus & 36.2 & $8.3 \mathrm{~mm}$ & $27 \pm 6$ & 2008 & ATCA & 0.3 & Potter et al. (2009) \\
\hline Torus & 44 & $6.8 \mathrm{~mm}$ & $40 \pm 2$ & 2011 & ATCA & 0.3 & Zanardo et al. (2013) \\
\hline Torus & 90 & $3.2 \mathrm{~mm}$ & $23.7 \pm 2.6$ & 2011 & ATCA & 0.7 & Lakićević et al. (2012b) \\
\hline Both & 110 & $2.8 \mathrm{~mm}$ & $27 \pm 3$ & 2012 & ALMA & $1^{\prime \prime} .3$ & This Letter \\
\hline Torus & 215 & $1.4 \mathrm{~mm}$ & $17 \pm 3$ & 2012 & ALMA & 0.7 & This Letter \\
\hline Ejecta & 215 & $1.4 \mathrm{~mm}$ & $<2$ & 2012 & ALMA & 0.7 & This Letter \\
\hline Torus & 345 & $870 \mu \mathrm{m}$ & $10 \pm 1.5$ & 2012 & ALMA & 0.5 & This Letter \\
\hline Ejecta & 345 & $870 \mu \mathrm{m}$ & $5 \pm 1$ & 2012 & ALMA & 0.5 & This Letter \\
\hline Torus & 680 & $440 \mu \mathrm{m}$ & $<7$ & 2012 & ALMA & 0.3 & This Letter \\
\hline Ejecta & 680 & $440 \mu \mathrm{m}$ & $50 \pm 15$ & 2012 & ALMA & 0.3 & This Letter \\
\hline Both & 860 & $350 \mu \mathrm{m}$ & $54 \pm 18$ & 2010 & Herschel & $24^{\prime \prime}$ & Matsuura et al. (2011) \\
\hline Both & 860 & $350 \mu \mathrm{m}$ & $44 \pm 7$ & 2011 & APEX & $8^{\prime \prime}$ & Lakićević et al. (2012a) \\
\hline Both & 1200 & $250 \mu \mathrm{m}$ & $123 \pm 13$ & 2010 & Herschel & $18^{\prime \prime}$ & Matsuura et al. (2011) \\
\hline Both & 1900 & $160 \mu \mathrm{m}$ & $125 \pm 42$ & 2010 & Herschel & 9.5 & Matsuura et al. (2011) \\
\hline Both & 3000 & $100 \mu \mathrm{m}$ & $54 \pm 18$ & 2010 & Herschel & $13^{\prime \prime} .5$ & Matsuura et al. (2011) \\
\hline
\end{tabular}

(Figure 3). This implies that that the carbon dust mass is much higher today than the $5 \times 10^{-4} M_{\odot}$ measured two years after the explosion (Wooden et al. 1993; Ercolano et al. 2007), and that within uncertainties nearly all of the $0.23 \pm 0.1 M_{\odot}$ of carbon (Thielemann et al. 1990; Woosley \& Heger 2007) released in the explosion is now in dust. The dust mass depends on opacity, temperature, and optical depth but is quite robustly constrained by the data. We use amorphous carbon opacity $\kappa=10$ and $3 \mathrm{~cm}^{2} \mathrm{~g}^{-1}$ at 450 and $870 \mu \mathrm{m}$, respectively (Rouleau \& Martin 1991). Values in the literature range from 2 to $10 \mathrm{~cm}^{2} \mathrm{~g}^{-1}$ at $450 \mu \mathrm{m}$ (e.g., Planck Collaboration et al. 2013; Jager et al. 1998); using a lower value would only raise the dust mass. Our analysis of $\mathrm{CO}$ emission observed with ALMA and Herschel finds $>0.01 M_{\odot}$ of CO in SN 1987A (Kamenetzky et al. 2013). Within uncertainties, the carbon in dust and $\mathrm{CO}$ does not yet exceed the nucleosynthetic yield, but as future observations refine the $\mathrm{CO}$ and dust masses, strong constraints may be placed on nucleosynthesis, chemistry, dust coagulation, or all three.

The temperature is quite well constrained by these data, and theoretical models predict a similar temperature. Cooling by adiabatic expansion and radiation is offset by heating from ${ }^{44} \mathrm{Ti}$ decay and by external X-ray heating from the shocks. The current gas temperature in the absence of X-ray heating is modeled to be 20-100 K (Fransson et al. 2013). Models predict 


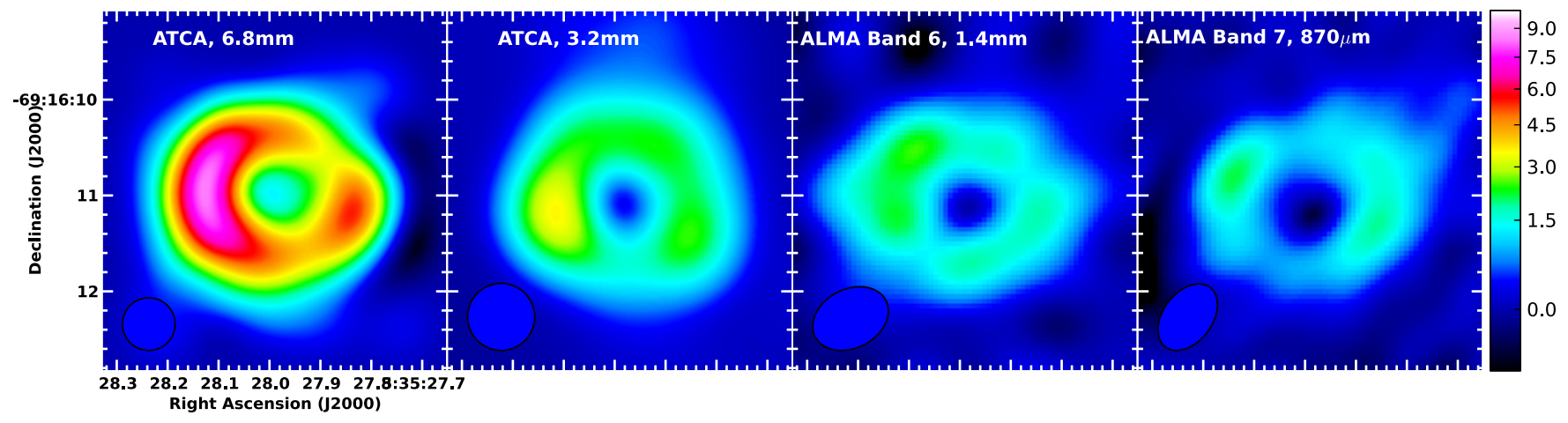

Figure 2. Emission from the synchrotron torus of SN 1987A as a function of wavelength, on the same intensity color scale. Emission from the inner ejecta has been subtracted to isolate the torus. The $6.8 \mathrm{~mm}$ ATCA image (Zanardo et al. 2013) has been smoothed to 0.55 beam similar to the ATCA $3.2 \mathrm{~mm}\left(0{ }^{\prime \prime} 7\right.$; Lakićević et al. 2012b) and ALMA images.

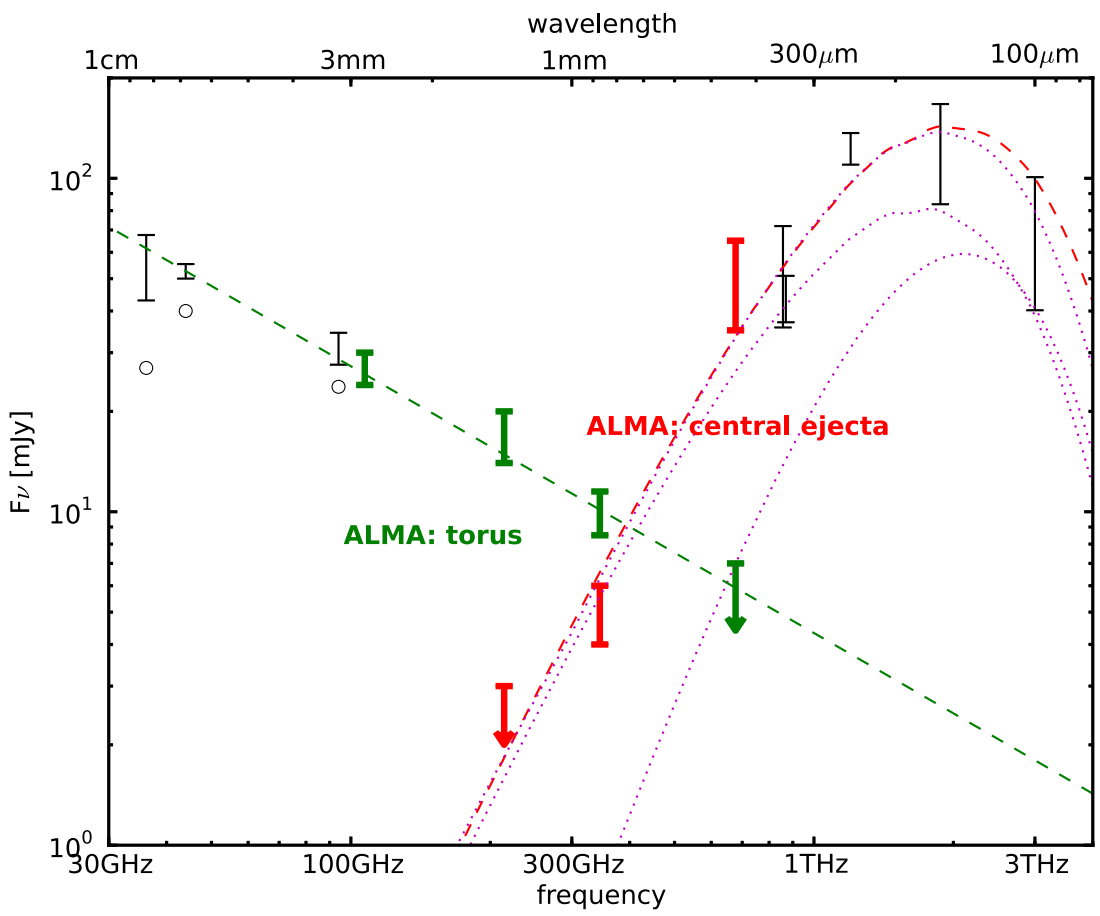

Figure 3. Spatially separated ALMA flux densities of the torus (green) and inner ejecta (red). Previous measurements are marked in black (Potter et al. 2009; Zanardo et al. 2013; Lakićević et al. 2012a, 2012b; Matsuura et al. 2011). Measurements at longer wavelengths dominated by shock emission have been scaled to the epoch of the ALMA observations according to the light curve $F_{v} \propto \mathrm{e}^{((t-5000) / 2231)}$ at $44 \mathrm{GHz}$ (Zanardo et al. 2010); the original flux densities at their epochs of observation are shown as open circles. The spectral energy distribution (SED) of the torus is a power law $F_{v} \propto v^{\alpha}$ with a single index $\alpha=-0.8 \pm 0.1$ (green dashed line). The SED of the inner ejecta is fit well by a model of dust emission-shown here is $0.23 M_{\odot}$ of amorphous carbon dust at $26 \mathrm{~K}$ (red dashed line), and a combination of amorphous carbon and silicate dust $\left(0.24 M_{\odot}\right.$ and $0.39 M_{\odot}$ respectively, both at $22 \mathrm{~K}$, two lower magenta dotted lines sum to the upper dotted line).

that significant X-rays do not yet penetrate inward as far as the $2 \times 10^{17} \mathrm{~cm}$ radius of the ALMA $450 \mu \mathrm{m}$ emission (Fransson et al. 2013). The compact and centrally peaked $450 \mu$ m emission supports this interpretation, since external heating would likely result in more limb-brightened or extended dust emission. Since the ionization fraction is below 1\% (Larsson et al. 2013), less than $\sim 40 \%$ of the X-ray flux will go into heating (Xu \& McCray 1991 ), and $<5 \%$ of the observed total flux $4.7 \times 10^{36} \mathrm{erg} \mathrm{s}^{-1}$ (Helder et al. 2013) is intercepted by the ejecta. Even if it did reach the dusty inner core, the energy deposition would be $<10 \%$ of the heating from ${ }^{44} \mathrm{Ti}$ decay (Jerkstrand et al. 2011). The best-fit dust mass $M_{d}$ scales approximately as $T_{d}^{-2}$, and $M_{d}>0.1 M_{\odot}$ for $T_{d}<50 \mathrm{~K}$.

The emission is optically thin at $450 \mu \mathrm{m}$, and $M_{d}$ is insensitive to unresolved clumpiness: If $0.23 M_{\odot}$ of dust uniformly filled a region the size of the ALMA emission, the peak surface density would be $0.02 \mathrm{~g} \mathrm{~cm}^{-2}$, with an optical depth $\tau_{450}<0.2$. If the dust is clumpy, each clump remains optically thin unless the filling fraction is less than $2 \%$; a filling fraction of $10 \%-20 \%$ was fitted to the infrared spectrum (Lucy et al. 1991; Ercolano et al. 2007) and consistent with the CO clump filling factor of 0.14 fitted to ALMA CO emission (Kamenetzky et al. 2013). Analytical formulae for radiative transfer in dense clumps (Városi \& Dwek 1999) indicate that the effective optical depth of the ensemble of clumps remains low over a very wide range of clump filling fraction and number.

The data can also be fit by a combination of carbonaceous and silicate $\left(\mathrm{Mg}_{2} \mathrm{SiO}_{4}\right)$ dust (Figure 3; dotted line), although the mass of carbonaceous dust cannot be significantly reduced because amorphous carbon has the highest submillimeter opacity $\kappa$ among minerologies similar to the interstellar medium, and eliminating it would require dust masses of other 


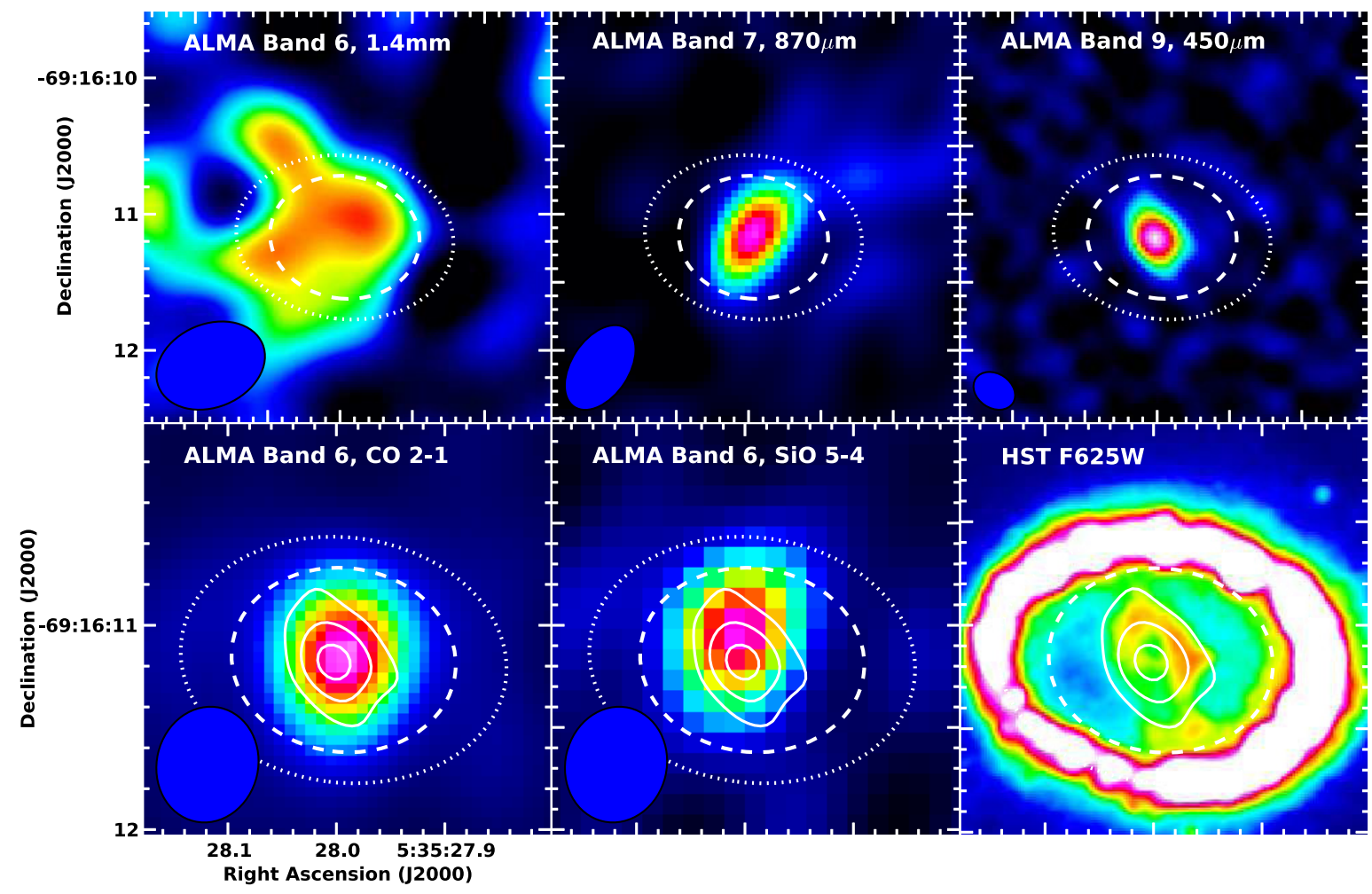

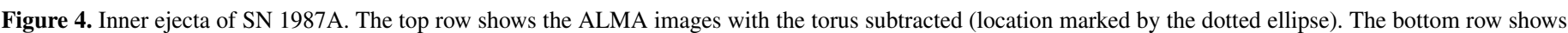

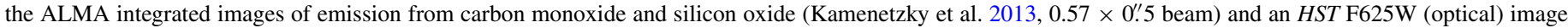

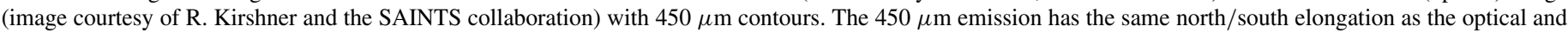

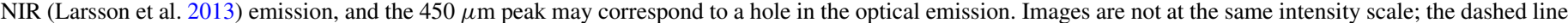
is the location of the reverse shock (Michael et al. 2003; France et al. 2010).

compositions larger than the available metal mass (the best fit using pure silicate is $4 M_{\odot}$ at $21 \mathrm{~K}$ ). Although these data do not strongly constrain the mass of silicate dust, it is unlikely that only carbon condensed in the SN 1987A, given the presence of large amounts of $\mathrm{Mg}$ and $\mathrm{Si}$ in the ejecta $(0.065 \pm 0.1$ and $0.19 \pm 0.1 M_{\odot}$, respectively) and the detection of $\mathrm{SiO}$ molecules at early epochs (Roche et al. 1991; Wooden et al. 1993). The absence of the $10 \mu \mathrm{m}$ feature during days $615-775$ puts a strong upper limit $(15 \%)$ on the fraction of dust which is silicates, but does not exclude the presence of some silicate dust during that epoch, since the ejecta could have been optically thick at that wavelength (Ercolano et al. 2007). The silicate mass could be much larger now, similar to the much larger carbon mass.

Models and observations show that core collapse supernovae like SN 1987A are highly inhomogeneous, with instabilities and radioactive energy deposition mixing the initially chemically stratified stellar interior into clumps with different compositions, macroscopically mixed throughout the ejecta volume (McCray 1993; Jerkstrand et al. 2011). The distribution of emission from different atoms, molecules, and dust not only reveals the chemistry of their formation, but a snapshot of the early supernova interior. The extent of dust emission corresponds well to line emission from $\mathrm{CO} 2-1$ and $\mathrm{SiO}$ 5-4 observed simultaneously with ALMA (Kamenetzky et al. 2013). The $1-2 \times 10^{17} \mathrm{~cm}$ radial extent corresponds to expansion velocities of $750-1400 \mathrm{~km} \mathrm{~s}^{-1}$, consistent with the $1250 \mathrm{~km} \mathrm{~s}^{-1}$ halfwidth of the CO emission (Kamenetzky et al. 2013), and within the commonly used maximum core expansion velocity of $\sim 2000 \mathrm{~km} \mathrm{~s}^{-1}$ (Jerkstrand et al. 2011). SiO is an important precursor to forming dust, so the relative distributions of dust and $\mathrm{SiO}$ in SN 1987A that ALMA will be able to measure with higher spatial resolution in the future will constrain formation theories.

In the last few years, the inner ejecta have developed a complex morphology in optical and infrared emission, including north-south elongation and a "hole" or fainter region in the center. The $450 \mu \mathrm{m}$ emission peak is coincident with the hole, and although the optical emission is likely limb-brightened due to external X-ray heating (Larsson et al. 2011), the dust we detect does have significant optical opacity. Smooth dust would have $\tau_{V}>1000$, and 100 dusty clumps filling $10 \%$ of the inner ejecta volume would have $\tau_{V} \sim 1.7$ (Városi \& Dwek 1999). (About 100 clumps were predicted by collapse simulations (Hammer et al. 2010) and fitted to observed optical lines (Jerkstrand et al. 2011)).

\section{CONCLUSIONS}

We have used the powerful resolution of ALMA to show clearly that prodigious amounts of dust have formed in the cold inner ejecta of SN 1987A. Our data suggests that nearly all of the carbon has condensed into dust, so condensation must be efficient. A large mass of clumpy dust was tentatively detected at $1.3 \mathrm{~mm}$ four years after the explosion (Biermann et al. 1992) - if this is the same dust then it formed quite rapidly. If dust production in other supernovae resembles that in SN 1987A, then core-collapse supernovae might contribute as much dust to galaxies as asymptotic giant branch stars. In the absence of grain destruction, dust in high-redshift galaxies can be explained with only $0.1 M_{\odot}$ produced per Type II supernova (Dwek et al. 2007). SN 1987A has the largest measured dust mass, but a few other remnants contain almost $0.1 M_{\odot}$ (Cassiopeia A, Barlow et al. 
2010; Crab Nebula, Gomez et al. 2012). However, dust formed in a supernova must survive passage through the reverse shock to be dispersed into the interstellar medium, and then also survive shock passages once dispersed (Jones \& Nuth 2011). The reverse shock in SN 1987A located with spatially resolved $\mathrm{H} \alpha$ spectra (Michael et al. 2003; France et al. 2010) has a minimum radius in the equatorial plane of $\sim 4 \times 10^{17} \mathrm{~cm}$, significantly larger than the $450 \mu \mathrm{m}$ emission (Figure 4). The new dust has not been significantly processed by the reverse shock. Models of dust destruction predict a wide range of survival fractions (Nozawa et al. 2007). If $0.23 M_{\odot}$ of dust is typically created in Type II supernovae, and $0.1 M_{\odot}$ passes through the reverse shock into the ISM, then Type II supernovae could dominate dust production in galaxies at all redshifts.

This Letter makes use of the following ALMA data: ADS/JAO.ALMA\#2011.0.00273.S (PI: Indebetouw). ALMA is a partnership of ESO (representing its member states), NSF (USA) and NINS (Japan), together with NRC (Canada) and NSC and ASIAA (Taiwan), in cooperation with the Republic of Chile. The Joint ALMA Observatory is operated by ESO, AUI/NRAO and NAOJ. The National Radio Astronomy Observatory is a facility of the National Science Foundation operated under cooperative agreement by Associated Universities, Inc. M.M. was supported by NASA NAG5-12595 and NASA/ADAP NNX13AE36G.

\section{REFERENCES}

Barlow, M. J., Krause, O., Swinyard, B. M., et al. 2010, A\&A, 518, L138 Biermann, P. L., Chini, R., Haslam, C. G. T., et al. 1992, A\&A, 255, L5 Bouchet, P., De Buizer, J. M., Suntzeff, N. B., et al. 2004, ApJ, 611, 394 Cherchneff, I., \& Dwek, E. 2010, ApJ, 713, 1

Crotts, A. P. S., \& Heathcote, S. R. 2000, ApJ, 528, 426

Dwek, E., Arendt, R. G., Bouchet, P., et al. 2010, ApJ, 722, 425

Dwek, E., Galliano, F., \& Jones, A. P. 2007, ApJ, 662, 927

Ellison, D. C., Berezhko, E. G., \& Baring, M. G. 2000, ApJ, 540, 292

Ercolano, B., Barlow, M. J., \& Sugerman, B. E. K. 2007, MNRAS, 375, 753
France, K., McCray, R., Heng, K., et al. 2010, Sci, 329, 1624

Fransson, C., Larsson, J., Spyromilio, J., et al. 2013, ApJ, 768, 88 Gaensler, B. M., \& Slane, P. O. 2006, ARA\&A, 44, 17

Gall, C., Hjorth, J., \& Andersen, A. C. 2011, A\&ARv, 19, 43

Gomez, H. L., Krause, O., Barlow, M. J., et al. 2012, ApJ, 760, 96

Gomez, H. L., Vlahakis, C., Stretch, C. M., et al. 2010, MNRAS, 401, L48

Hammer, N. J., Janka, H.-T., \& Müller, E. 2010, ApJ, 714, 1371

Helder, E. A., Broos, P. S., Dewey, D., et al. 2013, ApJ, 764, 11

Jager, C., Mutschke, H., \& Henning, T. 1998, A\&A, 332, 291

Jerkstrand, A., Fransson, C., \& Kozma, C. 2011, A\&A, 530, A45

Jones, A. P., \& Nuth, J. A. 2011, A\&A, 530, A44

Jura, M., Turner, J. L., Van Dyk, S., \& Knapp, G. R. 2000, ApJL, 528, L105

Kamenetzky, J., McCray, R., Indebetouw, R., et al. 2013, ApJL, 773, L34

Kirk, J. G., Duffy, P., \& Gallant, Y. A. 1996, A\&A, 314, 1010

Lakićević, M., van Loon, J. T., Stanke, T., De Breuck, C., \& Patat, F. 2012a, A\&A, 541, L1

Lakićević, M., Zanardo, G., van Loon, J. T., et al. 2012b, A\&A, 541, L2

Larsson, J., Fransson, C., Kjaer, K., et al. 2013, ApJ, 768, 89

Larsson, J., Fransson, C., Östlin, G., et al. 2011, Natur, 474, 484

Lucy, L. B., Danziger, I. J., Gouiffes, C., \& Bouchet, P. 1991, in Supernovae, ed. S. E. Woosley (New York: Springer-Verlag), 82

Matsuura, M., Dwek, E., Meixner, M., et al. 2011, Sci, 333, 1258

McCray, R. 1993, ARA\&A, 31, 175

Michael, E., McCray, R., Chevalier, R., et al. 2003, ApJ, 593, 809

Ng, C.-Y., Zanardo, G., Potter, T. M., et al. 2013, ApJ, 777, 131

Nozawa, T., Kozasa, T., Habe, A., et al. 2007, ApJ, 666, 955

Nozawa, T., Kozasa, T., Umeda, H., Maeda, K., \& Nomoto, K. 2003, ApJ, 598, 785

Planck Collaboration, Ade, P. A. R., Aghanim, N., et al. 2013, arXiv:1307.6815

Potter, T. M., Staveley-Smith, L., Ng, C.-Y., et al. 2009, ApJ, 705, 261

Racusin, J. L., Park, S., Zhekov, S., et al. 2009, ApJ, 703, 1752

Roche, P. F., Aitken, D. K., \& Smith, C. H. 1991, MNRAS, 252, 39P

Rouleau, F., \& Martin, P. G. 1991, ApJ, 377, 526

Thielemann, F.-K., Hashimoto, M.-A., \& Nomoto, K. 1990, ApJ, 349, 222

Todini, P., \& Ferrara, A. 2001, MNRAS, 325, 726

Tziamtzis, A., Lundqvist, P., Gröningsson, P., \& Nasoudi-Shoar, S. 2011, A\&A, 527, A35

Városi, F., \& Dwek, E. 1999, ApJ, 523, 265

Wooden, D. H., Rank, D. M., Bregman, J. D., et al. 1993, ApJS, 88, 477

Woosley, S. E., \& Heger, A. 2007, PhR, 442, 269

Xu, Y., \& McCray, R. 1991, ApJ, 375, 190

Zanardo, G., Staveley-Smith, L., Ball, L., et al. 2010, ApJ, 710, 1515

Zanardo, G., Staveley-Smith, L., Ng, C.-Y., et al. 2013, ApJ, 767, 98

Zhekov, S. A., McCray, R., Dewey, D., et al. 2009, ApJ, 692, 1190 